\title{
Verraten Sie nicht, wie billig Ihre Therapie ist
}

\author{
Chefredakteur Dr. med. Dirk Einecke
}

\section{PLACEBOEFFEKT}

\section{Teuer wirkt besser}

Wie gut ein Medikament wirkt, wird von der Erwartung des Patienten beeinflusst. Und diese Erwartungshaltung hängt offenbar auch vom Preis einer Therapie ab: In einer Studie wirkte ein Placebo stärker schmerzlindernd, wenn die Probanden glaubten, sie hätten ein 2,50\$ teures Opioid erhalten, als wenn man ihnen sagte, es handele sich um ein Opioid zu einem reduzierten Preis von $10 \mathrm{Ct}$ die Tablette.
Dieser Effekt spielt möglicherweise eine Rolle, wenn Patienten berichten, dass ein Generikum nicht so gut wirkt wie das Originalpräparat, so die Vermutung der Studienautoren. Ihre Empfehlung an die Ärzte: Sie sollten die geringen Kosten einer Therapie nicht betonen, sondern positive Erwartungen wecken, indem sie die Qualitäten eines Medikaments herausstellen.

JAMA 2008;299:1016-17

\section{„ER IST HALT EIN BISSCHEN KRÄFTIGER“}

\section{Übergewicht wird von Eltern oft ignoriert}

Eltern scheinen manchmal blind zu sein für die Körperfülle ihrer Kinder. Selbst bei stark übergewichtigen Kindern, die bereits wegen eines Typ-2-Diabetes in Behandlung sind, wird die Gewichtsproblematik oft ignoriert. So erfüllten in einer Studie $87 \%$ von 104 diabetischen Kindern die Kriterien einer Adipositas, aber nicht einmal die Hälfte der Eltern und der Kinder teilten diese Einschätzung. Eltern, die das Körpergewicht ihres Kindes für ,ungefähr richtig“ hielten, hatten zu 40\% adipöse Kinder. Diese Fehleinschätzung

\section{SCHLAGANFALL}

\section{Schlechter Ernährungs- zustand trübt die Prognose}

Patienten, die zum Zeitpunkt eines Schlaganfalls unterernährt sind, haben eine ungünstige Prognose, so das Ergebnis einer prospektiven Studie mit $131 \mathrm{~Pa}-$ tienten. $12 \%$ von ihnen befanden sich in einem mangelhaften Ernährungszustand, d. h. sie hatten in den Monaten vor dem Schlaganfall stark an Gewicht verloren oder wiesen erniedrigte Albuminund Transferrinspiegel auf. In dieser Gruppe kam es zu signifikant mehr Schlaganfallkomplikationen und einem insgesamt ungünstigeren klinischen Verlauf innerhalb der nächsten drei Monate als bei den wohlgenährten Patienten. Archives of Neurology 2008;65:39-43

\section{Nur ein bisschen pummelig?}

korrelierte mit einem besonders ungesunden Ernährungs- und Lebensstil. Ärzte sollten deswe-

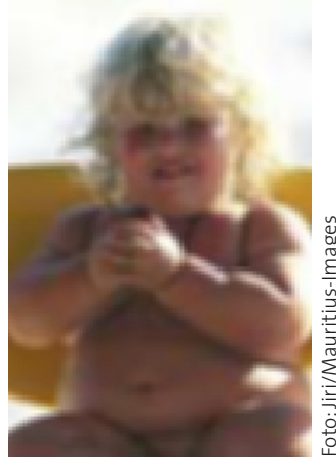

gen versuchen, Eltern und Kindern ein realistischeres Körperbild zu vermitteln. Diabetes Care 2008;31:227-29

\section{HORMONSUBSTITUTION ABGESETZT}

\section{Brustkrebsrisiko bleibt erhöht}

Frauen, die im Rahmen der Women's Health Initiative eine Hormonersatztherapie erhalten hatten, wiesen auch drei Jahre nach Abbruch dieses Studienarms ein höheres Brustkrebsrisiko auf als die Frauen der Placebogruppe. Von 15730 Frauen entwickelten 79 Frauen der Hormongruppe und 60 der Placebogruppe ein invasives Mammakarzinom. Andere Nebenwirkungen der Östrogen-Gestagen-Kombinationsbehandlung verschwanden hingegen schnell, besonders das erhöhte Herzinfarkt- und Thromboserisiko. Allerdings ließ sich auch der günstige Effekt auf den Knochen schon bald nicht mehr nachweisen. JAMA 2008;299:1036-45

\section{KARDIOVASKULÄRE NEBENWIRKUNGEN}

\section{Herkömmliche NSAR nicht sicherer als Coxibe}

Die kardiovaskulären Risiken der Coxibe wurden offenbar überschätzt und die der nicht selektiven NSAR unterschätzt. Einem aktuellen Review zufolge sind sie weitgehend identisch. Einzige Ausnahme: Naproxen. Dieses NSAR kann möglicherweise sogar das Herz-Kreislauf-Risiko senken. Allerdings schränken die Reviewautoren ihre Aussage dahingehend ein, dass alle Daten letztlich auf Studien zurückgehen, die nicht primär die kardiovaskulären Effekte untersucht haben. Bei der Wahl eines NSAR oder Coxibs sollte man außerdem auch die gastrointestinalen Risiken und die schmerzlindernde Wirkung berücksichtigen, betonen sie.

In einem vor kurzem veröffentlichten Lancet-Kommentar wird dieselbe Einschätzung vertreten. Die Autoren warnen vor der „immer populäreren Idee, dass traditionelle NSAR ein geringeres kardiovaskuläres Risiko haben als Coxibe".

J Cardiovasc Pharmacol Ther 2008;13:41-50; Lancet 2008; 371:270-73

\section{BRONCHIALKARZINOM}

\section{Vitamintabletten können das Krebsrisiko steigern}

Die Supplementation von Vitaminen bietet keinen Schutz vor Lungenkrebs. Sie kann sogar mit einer Zunahme von Erkrankungsfällen einhergehen, wie eine Studie mit 77000 Frauen und Männern ergeben hat. Über einen Zeitraum von vier Jahren entwickelten 521 von ihnen ein Bronchialkarzinom. Personen, die Vitaminpillen geschluckt hatten, waren davon genauso häufig betroffen wie andere. Ein erhöhtes Risiko zeigte sich erwartungsgemäß bei Rauchern, positiver Familienanamnese und höherem Alter. Überraschenderweise war aber auch die Einnahme von Vitamin E mit einem Risikoanstieg assoziiert. Die Einnahme von täglich $400 \mathrm{mg}$ über zehn Jahre erhöhte das Risiko um $28 \%$.

Am J Resp Cit Care Med 2008;177:524-30 